\title{
Efficacy of Using Neutrophil to Lymphocyte Ratio and Interleukin 6 as Outcome Predictors of Interventional Treatment of Hepatocellular Carcioma
}

\author{
Salama M El-Ghoneimy ${ }^{1}$, Amira M Soliman ${ }^{1}$, Mervat M Azab ${ }^{2}$, \\ Ahmed M El-Gebaly ${ }^{1}$, Sameh M Abdel Monem ${ }^{1}$ \\ ${ }^{1}$ Tropical Medicine Department, Faculty of Medicine, Zagazig University, Egypt \\ ${ }^{2}$ Clinical Pathology Department, Faculty of Medicine, Zagazig University, Egypt
}

Corresponding Author Ahmed M. El-Gebaly

Mobile: +20122554030 7

\section{E mail:} icegebo2011@yahoo.c om

Key words: HCC, interleukin-6, neutrophil , lymphocyte, ratio
Background and study aim: Hepatocellular carcinoma (HCC) is currently the fifth most common solid tumor worldwide and the third leading cause of cancer-related death. Percutaneous ethanol injection (PEI) and radiofrequency ablation (RFA) techniques became well-known procedures for controlling small HCC. Inflammation is a major contributing factor to carcinogenesis specially HCC. Inflammatory mediators including interleukin-6 (IL-6) and neutrophil to lymphocyte ratio (NLR) are good candidates to stimulate tumor growth and progression. The aim of this study was to evaluate validity of using NLR and IL-6 as predictors of outcome of interventional treatment for HCC.

Patients and methods: This study was conducted on 136 patients with 145 focal nodular HCCs of $5 \mathrm{~cm}$ or less between 2012 and 2015. They were divided into 2 groups, the first group included 72 patients whom had NLR less than 5 and the second group included 64 patients whom had NLR equal or more than 5. Patients underwent PEI or RFA. Clinical assessment, laboratory evaluation and triphasic CT studies were performed to all patients pre-treatment and at 1, 6 and 12 months post treatment. NLR and IL-6 level were performed to all patients pre treatment and at 6 months post treatment.

\section{INTRODUCTION}

Hepatocellular carcinoma (HCC) is the fifth most common form of cancer worldwide and the third most common cause of cancer-related deaths. HCC often occurs in the background of a cirrhotic liver [1]. Early detection
Results: The percentage of complete ablation is highly significantly higher in group I after 1 month but, there is no significant difference between outcome of treatment after 1 year in both groups one year after treatment. Post-procedural IL-6 showed highly significantly lower levels in patients whose focal lesions remain ablated till 1 year than in those who showed multifocal or local recurrence, while NLR did not show significant difference among these groups. Postprcedural IL-6 levels were highly significantly lower in survived than died cases, while NLR did not show significant differences between both groups at 1 year follow up. There was no significant difference in the post-procedural level of NLR or IL-6 between the group who was treated with ethanol injection and who was treated with radiofrequency ablation as regard the overall outcome at 1 year follow up.

Conclusions: Pre-procedural NLR is a good indicator of performance status, liver profile, hematological profile and diameter of focal HCC and also is a good predictor for short term outcome (1-6 months) and neither pre-procedural or post-procedural NLR are predictors of long term outcome or survival (1 year). Post-procedural IL-6 could be considered as a good predictor not only for survival, but also for efficacy of treatment.

strategies have increased the number of small HCC amenable to curative treatment [2]. Percutaneous ablation under ultrasound guidance is currently the best therapy for early-stage HCC when resection or liver transplantation is not possible [3]. 
Inflammation is a major contributing factor to carcinogenesis [4]. Cumulative evidence indicates that inflammatory diseases predispose to the development of different types of tumors [5]. HCC represents a classic case of inflammation linked cancer and chemically or genetically induced HCC depends on inflammatory signaling [4]. Indeed, HCC almost always develops on a background of chronic liver injury including chronic hepatitis and cirrhosis, conditions regarded as preneoplastic stages [6].

Inflammatory mediators including interleukin-6 (IL-6) and neutrophil to lymphocyte ratio (NLR) are good candidates to stimulate tumor growth and progression [7-10].

NLR is obtained by dividing the absolute neutrophil count by the absolute lymphocyte count. NLR more than or equal to 5 can be considered a valid cutoff $[9,10]$. Patients with elevated NLR have a relative lymphocytopenia and neutrophilic leukocytosis which denote that the balance is tipped in favor of protumor inflammatory response and is associated with poor oncologic outcome [11]. That fact was observed in many tumors [12-15].

Elevated NLR may correlate with a worse prognosis in patients with HCC who underwent any radiological intervention [16]. Preoperative NLR more than or equal to 5 was an adverse predictor of disease free and overall survival for patients undergoing hepatic resection for HCC [10]. Increased NLR significantly increases the risk of HCC recurrence and recipient death in patients undergoing transplantation for HCC [17].

IL-6 is essential to trigger hepatocyte proliferation, liver regeneration and survival after partial hepatectomy [18]. IL-6 was postulated to correlate with the stage of liver cirrhosis $[\mathbf{1 9 , 2 0}]$. IL-6 has both differentiation and growth promoting effects for target cells. It has also been hypothesized that activation of IL- 6 gene might trigger initial events leading to oncogenic transformation. IL-6 concentrations are also increased in patients with HCC relative to normal subjects $[\mathbf{1 9 , 2 0}]$. There was a significant increase in serum IL-6 concentration of HCC patients as compared to cirrhotic patients. Serum IL-6 level was positively correlated with serum $\alpha$-fetoprotein [20].

The aim of this study was to evaluate validity of using NLR and IL-6 as predictors of outcome of interventional treatment for HCC.

\section{PATIENTS AND METHODS}

This study was conducted in the Tropical Medicine in cooperation with Radiotherapy and Clinical Pathology Departments, Faculty of Medicine, Zagazig University, Egypt, during period from December 2012 to February 2015 and included 136 patients presented with 145 focal HCC lesions who underwent Percutaneous Ethanol Injection or Radio Frequency Ablation therapy. Sample size estimation was performed by the Institutional Review Board (IRB). The patients were divided into 2 groups:

Group I: Contained 72 patients with 75 focal lesions (69 patients had single lesion and 3 patients each had 2 lesions). These patients had NLR less than 5.

Group II: Contained 64 patients with 70 focal lesions (58 patients had single lesion and 6 patients each had 2 lesions). These patients had NLR equal or more than 5 .

The diagnosis of HCC in a patient with hepatic focal lesion was based on triphasic CT scan showing typical criteria for HCC (early enhancement during arterial phase followed by washout of contrast in porto-venous and delayed phases).

All patients met the enrolment criteria: (i) patients with single focal lesion $\leq 5 \mathrm{~cm}$ or three lesions each $\leq 3 \mathrm{~cm}$, (ii) liver cirrhosis of ChildPugh class A or B or non cirrhotic, (iii) platelet counts $>50000 \mathrm{~mm}^{3}$, (iv) prothrombin concentration $>60 \%$ or INR $<1.5$, (v) adequate kidney functions (serum creatinine $\leq 2 \mathrm{mg} / \mathrm{dl}$ ). (vi) absence or controlable ascites, (vii) performance status 0-2, (viii) no history of hepatic encephalopathy, (ix) no evidence of extrahepatic metastasis, (x) no evidence of portal vein thrombosis and (xi) no history of operation, chemotherapy, and ablative therapy for the lesions.

Patients with Child-Pugh class C, vascular invasion, extrahepatic metastasis and the patients with coexistent haematological disorders or known active infection (affecting total or differential leucocytic count) were excluded.

A written informed consent was taken from all included patients, and the ethical committee of the university has accepted the study.

\section{Pretreatment assessment}

Pre treatment assessment of all patients was done by full history taking, thorough clinical examination, laboratory investigations including liver function, 
kidney function, Complete blood count including total and differential WBC counts, $\alpha$ fetoprotein, serological markers for $\mathrm{HCV}$ and $\mathrm{HBV}$, NLR (was calculated from the differential count by dividing the absolute neutrophil count by the absolute lymphocyte count) and IL-6 measurement. Radiological examination including ultrasound and triphasic CT study.

\section{RESULTS}

Table (1): Comparison between studied groups as regard outcome of treatment after 1 month

\begin{tabular}{|c|c|c|c|c|c|c|c|}
\hline \multirow{2}{*}{$\begin{array}{c}\text { Outcome of procedure } \\
\text { after } 1 \text { month }\end{array}$} & \multicolumn{2}{|c|}{ Group I $(n=72)$} & \multicolumn{2}{|c|}{ Group II (n=64) } & \multirow{2}{*}{$\chi^{2}$} & \multirow[b]{2}{*}{$\mathbf{p}$} & \multirow{2}{*}{ Sig. } \\
\hline & No & $\%$ & No & $\%$ & & & \\
\hline Complete ablation & 46 & $63.9 \%$ & 21 & $32.8 \%$ & \multirow{2}{*}{13.091} & \multirow{2}{*}{$<0.001$} & \multirow{2}{*}{ (HS) } \\
\hline Partial ablation & 26 & $36.1 \%$ & 43 & $67.2 \%$ & & & \\
\hline
\end{tabular}

In our study the percentage of complete ablation is highly significantly higher in group I while, the percentage of partial ablation is highly significantly higher in group II.

In our study the percentage of stationary outcome is significantly higher in group I. Otherwise; other outcomes do not show significant differences between both groups as shown in table (2).

Table (2): Follow up of the patients with complete ablation in both groups 6 months after procedures

\begin{tabular}{|c|c|c|c|c|c|c|c|}
\hline \multirow{2}{*}{$\begin{array}{l}\text { Outcome of procedures } \\
\text { after } 6 \text { months }\end{array}$} & \multicolumn{2}{|c|}{$\begin{array}{c}\text { Group I } \\
(n=46)\end{array}$} & \multicolumn{2}{|c|}{$\begin{array}{c}\text { Group II } \\
(\mathbf{n}=21)\end{array}$} & \multirow[t]{2}{*}{$\chi^{2}$} & \multirow[t]{2}{*}{$\mathbf{p}$} & \multirow[t]{2}{*}{ Sig. } \\
\hline & No & $\%$ & No & $\%$ & & & \\
\hline Stationary & 33 & 71.7 & 9 & 42.9 & 5.142 & 0.023 & (S) \\
\hline Multifocal & 5 & 10.9 & 4 & 19 & 0.829 & 0.362 & $(\mathrm{NS})$ \\
\hline Local recurrence & 6 & 13 & 5 & 23.8 & 1.218 & 0.270 & $(\mathrm{NS})$ \\
\hline Died & 2 & 4.3 & 3 & 14.3 & 2.062 & 0.151 & $\overline{(\mathrm{NS})}$ \\
\hline
\end{tabular}

Table (3): Correlation between preprocedural NLR and selected study parameters

\begin{tabular}{|l|c|c|}
\hline \multicolumn{2}{|c|}{ Parameters } & \multicolumn{2}{c|}{ Preprocedural NLR } \\
\cline { 2 - 3 } & $\mathbf{~ r ~}$ & p \\
\hline Bilirubin $(\mathrm{mg} / \mathrm{dL})$ & +0.214 & $0.012(\mathrm{~S})$ \\
\hline PT $(\mathrm{sec})$ & +0.197 & $0.022(\mathrm{~S})$ \\
\hline Hemoglobin $(\mathrm{g} / \mathrm{dL})$ & -0.452 & $<0.001(\mathrm{HS})$ \\
\hline Platelet count $\left(\mathrm{x} 10^{3} / \mathrm{mm} 3\right)$ & -0.455 & $<0.001(\mathrm{HS})$ \\
\hline Age $($ years) & +0.519 & $<0.001(\mathrm{HS})$ \\
\hline Diameter of focal lesion $(\mathrm{cm})$ & +0.277 & $0.001(\mathrm{HS})$ \\
\hline
\end{tabular}

There is a significant direct correlation between the mean pre-procedural NLR with bilirubin and prothrombin time and a highly significant direct correlation with age and diameter of focal lesion and a highly significant indirect correlation with hemoglobin and platelet count.

Table (4): Relation between post-procedural IL-6 and outcome after 1 year

\begin{tabular}{|l|c|c|c|c|c|c|}
\hline & $\begin{array}{c}\text { Stationary } \\
(\mathbf{n = 3 5})\end{array}$ & $\begin{array}{c}\text { Multifocal } \\
(\mathbf{n = 1 1})\end{array}$ & $\begin{array}{c}\text { Local } \\
\text { recurrence } \\
(\mathbf{n = 1 0})\end{array}$ & Test & p & Sig. \\
\hline IL-6 & & & & KW & & \\
\hline Mean & 83.29 & 192.64 & 141.5 & \multirow{2}{*}{13.341} & 0.001 & $(\mathrm{HS})$ \\
\hline SD & 90.21 & 153.30 & 135.19 & & \\
\hline
\end{tabular}

There is high significant difference between stationary, multifocal and local recurrence groups as outcome after one year as regard post-procedural IL-6. 
Table (5): Relation between postprocedural IL-6 and survival after 1 year

\begin{tabular}{|l|c|c|c|c|c|}
\hline \multicolumn{2}{|c|}{ Survive (n=116) } & Died (n=20) & MW & p & Sig. \\
\hline IL-6 & $102.91 \pm 106.12$ & $237.5 \pm 121.22$ & & & \\
\hline Mean \pm SD & $70(3-437)$ & $215.5(65-437)$ & -4.727 & $<0.001$ & \multirow{2}{*}{ (HS) } \\
\hline Median (Range) &
\end{tabular}

There is high significant difference between survived and died groups after one year as regard postprocedural IL-6.

\section{DISCUSSION}

Till the time this study is planned for in October 2012, many studies had been published to evaluate validity of using either NLR or IL-6 as predictors of outcome of interventional treatment for HCC but - to our knowledge- no studies including both NLR and IL6 in the same study.

Jang and collegues showed that IL-6 was the most significant cytokine predictive of HCC survival. A high serum IL-6 level appeared to reflect the tumor burden, because it was significantly associated with tumor size, stage and aggressiveness such as portal vein invasion and metastasis [21]. $\mathrm{Kim}$ and collegues measured the level of 13 member of cytokine family in HCC patients and found that Among the 13 cytokines tested in this study, IL-6 was most strongly related to tumor characteristics including tumor size, number, and the presence of metastasis suggesting a significant role of IL-6 in HCC progression [22].

In this study, the patients in group II (NLR $\geq 5$ ) are highly significantly older than group I (NLR $<5)$. Also there is a highly significant direct correlation between the mean preprocedural NLR and age and this is not in agreement with Huang et al. [16] who found no difference as regard age while, there is no significant difference as regard sex between both groups in agreement with $\mathrm{Fu}$ et al. [31]. There is no significant correlation between pre-procedural IL-6 and age in agreement with many researchers $[\mathbf{2 4 , 2 5 , 3 0 ]}$.

As regard viral hepatitis profile, the mean preprocedural NLR was significantly higher in patients who are infected by both hepatitis B and C viruses. Between both groups there was no significant difference in the percentage distribution of viral markers in agreement with Liao et al. [23] but not in agreement with Huang et al.[16] who studied 145 patients, most of them was HBs $\mathrm{Ag}$ +ve. IL-6 did not show any significant differences between groups as regard viral hepatitis profile in agreement with Chau et al. and Pang et al. $[24,25]$.
As regard clinical presentation right hypochondrial pain was the most frequent symptom in both groups while, lower limb edema and history of ascites were present in group II only with significant difference in agreement with Gomez et al. [10] but not in agreement with McNally et al[27] who found no significant difference as regard presence of ascites between both groups. There was no significant difference as regard ultrasonographic findings between both groups in agreement with Pinato et al. [28].

The mean diameters of focal lesions were highly significantly larger in group II than group I. There was a highly significant direct correlation between the mean preprocedural NLR and diameter of focal lesion in agreement with many researchers $[23,31,36]$ but not in agreement with Huang et al and Motomuraet al. $[\mathbf{1 6 , 2 9}]$ because they studied 158 patients, 101 cases of them had received previous lines of treatment for HCC before the beginning of the study.

The explanation of that association is unclear. One possible explanation is that patients with a high NLR usually have an enhanced neutrophil response. Circulating neutrophils could produce and secrete pro-angiogenetic factors, including VEGF [37], interleukin- 8 (IL-8) [38] and MMP [39] which may promote tumor growth. Another explanation is that the host's immune response to tumors depends on lymphocytes, and patients with a high NLR have relative lymphocytopenia and neutrocytosis. Lymphocyte-mediated antitumor immune reaction is attenuated in these patients. On the other hand, neutrocytosis can inhibit the cytolytic activity of lymphocytes, natural killer cells, and activated $\mathrm{T}$ cells. Thus, both lymphocytopenia and neutrocytosis may be important factors in tumor progression.

There was no significant correlation between the mean preprocedural IL-6 and diameter of focal lesions. Also IL-6 did not show significant difference in its level between large and small focal lesions in agreement with Parasole et al [30] but not in agreement with Jang et al. and 
Pang et al. [21,25] who proved that high levels of serum IL-6 were associated with larger tumor diameters in HCC patients and they considered the high serum IL-6 levels as a novel tumor marker for HCC.

There was no significant difference as regard number of focal lesions in both groups. There were no significant differences in the mean preprocedural NLR and the number of focal lesions in agreement with Motomura et al. [29] and not in agreement with McNally et al. [27] who studied 103 HCC patients with multifocal lesions and most of them had more than 3 focal lesions. Also there was no significant differences in the mean preprocedural IL-6 and the number of focal lesions in agreement with many researchers $[\mathbf{2 1}, \mathbf{2 4 , 2 5 , 3 2 ]}$.

There was no significant difference in the percentage distribution of Child-Pugh classes between both groups before procedure. After procedure there was highly significant downgrading in ChildPugh classes in patients of group II than group I. This downgrading could be due to the inflammatory response induced by the locoregional treatment which is more prominent in group II or due to the natural history of cirrhosis itself. There was no significant correlation between the mean preprocedural NLR and Child-Pugh classes in agreement with some researchers $[\mathbf{2 8 , 3 5 ]}$ but not in agreement with $\mathrm{Fu}$ et al. [31] who studied 282 Chinese patients with HBV-associated HCC after radical hepatectomy. Hence, the etiology of HCC may affect the predictive ability of NLR.

There was no significant correlation between the mean preprocedural IL-6 and Child-Pugh classes in agreement with Parasole et al. [30] and not in agreement with Kim et al[22] who measured the level of 13 member of cytokine family in Korean HCC patients and found significant association between IL-6 and Child-Pugh class.

The liver profile tests were more deranged in patients with elevated NLR who showed more derangement after locoregional treatment than patients with normal NLR. Patients with elevated NLR showed also elevation in serum creatinine after locoregional treatment which is absent in patients with normal NLR.

Although the impaired synthetic function accompanying chronic liver disease is a main determinant of reduced serum albumin, this reduction also reflects subclinical inflammatory response and malnutrition. These overlapping conditions affect the prognosis of cancer patients.
Hepatic albumin biosynthesis is downregulated by proinflammatory stimuli as part of a negative acute phase reaction, in patients with malignancy [40]. This is obvious in this study where serum albumin significantly reduced after ethanol injection or radiofrequency ablation in both groups. The cell death that occur after these maneuvers leads to production of macromolecules called damage associated molecular patterns (DAMPs) that trigger the production of cytokines and other proteins that regulate and amplify the inflammatory response with its consequences [4].

There was a significant direct correlation between the mean preprocedural NLR and bilirubin and prothrombin time. IL-6 did not show any correlation with these laboratory results in agreement with Chau et al. and Pang et al. [24,25].

Hemoglobin and platelet count were highly significantly lower in group II before and after procedure while, after procedure there were highly significant lowering in hemoglobin, platelet count and white blood cells count in both groups. There was a highly significant indirect correlation between the mean preprocedural NLR and hemoglobin concentration and platelet count. That was not in agreement with Fu et al. [31].

In this study alpha fetoprotein showed highly significant decrease in group I and significant decrease in group II after procedure while no significant difference in its level between both groups either before or after procedures. Neither preprocedural NLR or IL-6 showed any correlation with alpha fetoprotein in agreement with Motomura et al. and Parasole et al. [29,30] but not in agreement with Wang et al [36] who studies 101 patients, most of them had larger focal lesion from 5 up to 8 centimeters and found direct correlation between NLR and alpha fetoprotein.

There was a highly significant direct correlation between the mean preprocedural NLR and the mean postprocedural NLR in agreement with Huang et al. [16] but not in agreement with McNally et al. [27] who studied 103 HCC patients underwent TACE. This may be attributed to the effect of TACE as different procedure on NLR.

After procedure, IL-6 increased in group I and decreased in group II insignificantly and there was highly significant difference in the level of postprocedural IL-6 between both groups in agreement with Chau et al.[24] but not in 
agreement with Erinjeri et al. [34]. Both studies showed change in the level of IL-6 but no study of them classified patients from the start according to NLR.

The percentage of complete ablation was highly significantly higher in group I while, the percentage of partial ablation was highly significantly higher in group II in agreement with Fu et al.[31] but not in agreement with Sullivan et al. [35] who studied lower number of cases ( 75 HCC patients) who underwent different procedures as resection, surgery or transplantation. The mean preprocedural NLR was highly significantly higher in patients with partial ablation than patients with complete ablation in agreement with Liao et al. [23] but not in agreement with McNally et al. [27] who studied 103 HCC patients underwent TACE and found no statistically significant difference in the mean preprocedural NLR between the partial and complete ablation. IL-6 did not show significant difference between patients with partial ablation or patients with complete ablation in agreement with Chau et al. and Pang et al. [24,25].

The percentage of stationary outcome was significantly higher in group I than group II 6 months after procedure while, one year after procedure there was no significant difference between outcome of complete ablation in both groups. Also follow up of all patients in both groups one year after procedures showed that there was no significant difference as regard outcome of procedures in both groups. The mean preprocedural NLR levels did not show significant difference between groups with different outcomes not in agreement with Liao et al.[23] who studied $256 \mathrm{HCC}$ patients underwent surgery with longer duration of follow up. IL-6 levels did not show any significant difference between groups with different outcomes in agreement with Pang et al.[25].

There was no significant difference in one year survival between both groups. Also there was no significant difference in the mean preprocedural NLR between the survived and died groups in agreement with $\mathrm{Fu}$ et al.[31] but not in agreement with Pinato et al.[28] who performed a meta analysis of $578 \mathrm{HCC}$ patients underwent different lines of treatment as surgery, interventional radiology, sorafenib or chemotherapy. Also, there was no significant difference in the mean preprocedural IL-6 between the survived and died groups in agreement with
Chau et al.and Parasole et al. [24,30] but not in agreement with Pang et al.[25] who studied only 80 patients underwent resection. Also not in agreement with Jang et al.[21] who studied 110 patient underwent TACE.

In this study, the measurement of NLR and IL-6 was done after six months after procedure in order to limit the influence of post procedure inflammation on the results. A study made by Erinjeri et al. [34] showed that percutaneous thermal ablation in HCC patients invokes an inflammatory response that is characterized by elevation in the plasma levels of specific cytokines. IL- 6 and IL-10 show the most robust increases in plasma levels within 48 hours of ablation. Also they showed that in patients undergoing image-guided ablation, heat-based ablation techniques showed 3.5 fold increases in IL-6 levels compared with a 54 fold increase with cryoablation. Some researchers showed that after open surgical radiofrequency ablation of hepatic malignancy, IL-6 levels increased 4 fold and after open hepatic cryoablation, IL-6 levels increased more than 100 fold $[\mathbf{4 1 , 4 2 ]}$.

The postprocedural IL-6 showed highly significant difference among patients with different outcomes at one year follow up while, postprocedural NLR did not show any significant difference among different outcomes or survived and died groups. So, postprocedural IL-6 could be considered as a good predictor not only for survival, but also for efficacy of treatment in agreement with Wong et al.[43] who proved that patients with hepatitis B and a high plasma level of IL-6 have HCC free survival three times shorter than patients with low IL-6 levels, even after controlling for age, gender, cirrhosis, use of antiviral treatments, hepatitis B virus DNA, and peak alanine aminotransferase levels. This is not in agreement with Chau et al. [24] who found no significant difference in the outcome between patients with high or low levels of serum IL-6 and with Parasole et al. [30] who failed to confirm that serum IL-6 correlates with HCC stage or prognosis.

Theoretically, NLR and IL-6 were expected to decrease after procedure because necrotic tumor cells can generate a permanent immunogenic source of tumor antigens (in situ tumor vaccine) for the induction of antitumor immunity. Previous reports described spontaneous regression of pulmonary metastases from renal cell carcinoma after thermal ablation of primary tumor Sanchez- 
Ortiz et al. [44] or regression of multiple pulmonary metastases after ablation of single metastasis [45].

Induced immune response after interventional procedure is mostly weak, and not sufficient for the complete eradication of established tumors or durable prevention of disease progression, but it could produce a favorable effect on the outcome and survival Dan et al. [46] but in some patients, the balance between host inflammatory response and immune response is tipped in favor of protumor inflammatory response where IL-6 remain high or increased after interventional treatment and predicts poor oncologic outcome. In this group of patients, it is reasonable to manipulate the systemic inflammatory response through targeted anti-inflammatory mediators such as IL-6 blocking antibodies Chua et al.[26]. There was no significant difference in the postprocedural level of NLR or IL-6 between the group who was treated with ethanol injection and who was treated with radiofrequency ablation as regard the overall outcome at 1 year follow up.

The conflict between results of different studies of NLR or IL-6 may be attributed to differences in age, performance status, Child-Pugh classes as some studies included Child $\mathrm{C}$, different races and population, different criteria of selection of HCC patients as presence of metastasis, portal vein invasion, larger diameter of focal lesions or different alpha fetoprotein levels, different predisposing factors of HCC including different serology of viral hepatitis, also use of different lines of treatment as percutaneous ablation, transarterial chemoembolization, systemic chemotherapy, hepatic resection, sorafenib, liver transplantation or mixed lines of treatment.

\section{Conflict of Interest: None}

\section{Funding:None.}

Ethical approval: The protocol of the study was approved by the ethical committee of Faculty of Medicine, Zagazig University. Informed consents were obtained from all patients.

\section{REFERENCES}

1- El- Serag H. B , Rudolph K.L . Hepatocellular carcinoma: epidemiology and molecular carcinogenesis. Gastroenterology 2007; 132 (7): 2557- 76 .

2- Bosch FX, Ribes J, Diaz M, Cleries R. Primary liver cancer: worldwide incidence and trends. Gastroenterology 2004; 127: S5-S16.
3- Germani G, Pleguezuelo M, Gurusamy K, Meyer T, Isgro G, Burroughs AK. Clinical outcomes of radiofrequency ablation, percutaneous alcohol and acetic acid injection for hepatocellular carcinoma: a meta-analysis. J Hepatol 2010, 52: 380-388.

4- Karin M. Nuclear factor- $\kappa \mathrm{B}$ in cancer development and progression. Nature 2006; 441: 431-436.

5- Kuper H., Adami H. , Trichopoulos, D. Infections as a major preventable cause of human cancer. $J$ Intern Med 2000; 248: 171-83.

6- Laurent-Puig P , Zucman-Rossi J.Genetics of hepatocellular tumors. Oncogene 2006; 25: 377886

7- Lin W , Karin M . A cytokine-mediated link between innate immunity, inflammation and cancer. J Clin Invest2007; 117: 1175- 83.

8- Fukata M , Abreu MT . Role of Toll-like receptors in gastrointestinal malignancies. Oncogene 2008 2008; 27: 234- 43 .

9- Halazun KJ, Aldoori A, Malik HZ, Al-Mukhtar A, Prasad KR, Toogood GJ, LodgeJP. Elevated preoperative neutrophil to lymphocyte ratio predicts survival following hepatic resection for colorectal liver metastases. Europ J Surg Oncol 2008; 34 (1): 55- 60.

10- Gomez D, Farid S , Malik H . Pre-operative neutrophil to lymphocyte ratio as a prognostic predictor after curative resection for hepatocellular carcinoma. World J Surg 2008; 32: 1757- 62.

11- Bertuzzo VR, Cescon M. , Ravaioli M. Analysis of factors affecting recurrence of hepatocellular carcinoma after liver transplantation with a special focus on inflammation markers. Transplantation 2011; 91: 1279-85.

12- Ding PR, An X, Zhang RX, Fang YJ, Li LR, $\mathrm{Chen} \mathrm{G}$,et al. Elevated preoperative neutrophil to lymphocyte ratio predicts risk of recurrence following curative resection for stage IIA colon cancer. Int J Colorectal Disease 2010; 25 (12): 1427- 33.

13-Kao SC, Pavlakis N, Harvie R . High blood neutrophil to lymphocyte ratio is an indicator of poor prognosis in malignant mesothelioma patients undergoing systemic therapy. Clin Cancer Res 2010; 16:5805-13.

14-Porrata L, Ristow K, Habermann T, Inwards DJ, Micallef IN, Markovic SN. Predicting survival for diffuse large B-cell lymphoma patients using baseline neutrophil/ lymphocyte ratio. Am. J. Hematol 2010; 85: 896-9.

15- Sharaiha R Z, Halazun K, Mirza F. Elevated preoperative neutrophil: lymphocyte ratio as a predictor of postoperative disease recurrence in esophageal cancer. Ann Surg Oncol 2011; 20(4): 619-35. 
16-Huang ZL,Luo J, Chen M, Li JQ, Shi M. Blood neutrophil to lymphocyte ratio predicts survival in patients with unresectable hepatocellular carcinoma undergoing transarterial chemoembolization. $J$ Vasc Interv Radiol 2011; 22: 702-9.

17- Halazun KJ, Hardy M, Rana A . Negative impact of neutrophil lymphocyte ratio on outcome after liver transplantation for hepato-cellular carcinoma. Ann Surg 2009; 250: 141- 51.

18- Fausto N, Campbell J, Riehle K . Liver regeneration. Hepatology 2006; 43: S45-S53.

19- Soresi M, Giannitrapani L, D’Antona F, Florena AM, La Spada E, Terranova A, et al. Interleukin-6 and its soluble receptor in patients with liver cirrhosis and hepatocellular carcinoma. World $J$ Gastroenterol 2006; 12: 2563-8.

20- Porta C, De Amici, M, Quaglini S, Paglino C, Tagliani F, Boncimino A et al. Circulating interleukin-6 as a tumor marker for hepatocellular carcinoma. Ann Oncol 2008; 19: 353- 58.

21- Jang JW, Byong SO, Jung HK, You CR, Chung KW, Kay CS, Jung HS. Serum interleukin-6 and C-reactive protein as a prognostic indicator in hepatocellular carcinoma. Cytokine 2012; 60: 68693.

22- Kim MJ, Jeong, WJ, Byong, SO, Kwon JH, Chung KW, Jung HS, et al. Change in inflammatory cytokine profiles after transarterial chemotherapy in patients with hepato-cellular carcinoma. Cytokine 2013b; 64: 516- 22.

23- Liao W, Zhang J, Zhu Q, Qin L, Yao W, Lei B, et al. Preoperative Neutrophil-to-Lymphocyte Ratio as a new prognostic marker in hepatocellular Carcinoma after curative resection. Translational Oncology2014; (7): 248- 55.

24- Chau GY, Chew-Wun W, Wing-Yiu, L, Chang TJ, Kao HL, Wu LH et al. Serum Interleukin-10 But Not Interleukin-6 Is Related to Clinical Outcome in Patients With Resectable Hepatocellular Carcinoma. Annals of Surgery 2000; (231)4:, 552-558.

25- Pang XH, Zhang JP, Zhang YJ, Yan J, Pei XQ, Zhang YQ et al. Preoperative Levels of Serum Interleukin-6 in Patients with Hepatocellular Carcinoma. Hepato-Gastroenterology 2011 ; 58:1687- 93.

26- Chua W, Charles KA, Baracos, VE; Clarke SJ. Neutrophil/lymphocyte ratio predicts chemotherapy outcomes in patients with advanced colorectal cancer. Br. J. Cancer 2011; 104: 1288-95.

27- McNally ME, Martinez A, Khabiri H, Guy G, Michaels AJ, Hanje Jet al. Inflammatory Markers are Associated with Outcome in Patients with Unresectable Hepato-cellular Carcinoma Undergoing Transarterial Chemoembolization. Ann Surg Oncol 2013 ; 20: 923-8.
28- Pinato DJ, Stebbing J Ishizuka, M, Khan SA, Wasan HS, North BVet al. A novel and validated prognostic index in hepatocellular carcinoma: The inflammation based index (IBI). Journal of Hepatology 2012; 57 : 1013- 20.

29- Motomura T, Shirabe K, Mano, Y; Muto J, Toshima T, Umemoto $\mathrm{Y}$ et al. Neutrophillymphocyte ratio reflects hepatocellular carcinoma recurrence after liver transplantation via inflammatory microenvironment. Journal of Hepatology 2013; 58 :j 58- 64.

30- Parasole R, Izzo F, Perrone F, Pignata S, Galati MG, Leonardi E et al. Prognostic Value of Serum Biological Markers in Patients with Hepatocellular Carcinoma. Clin Cancer Res 2001; 7: 3504- 09.

31- Fu SJ, Shun-Li S, Shao-Qiang L, Hua YP, Hu WJ, Liang LJet al. Prognostic value of preoperative peripheral neutrophilto-lymphocyte ratio in patients with $\mathrm{HBV}$-associated hepatocellular carcinoma after radical hepatectomy. Med Oncol 2013; 30: 721- 24.

32- Moustafa EF, Galal GM, Hassany SM et al. Serum interleukin-6 (IL-6), vascular endothelial growth factor (VEGF), and VEGF/ platelets ratio as markers for hepatocelluar carcinoma. Life Sci J 2012; 9 (2): 930- 8.

33- Metwaly HA, Al- Gayyar MH, Eletreby S, Ebrahim MA, El-Shishtawy MM. Relevance of Serum Levels of Interleukin-6 and Syndecan-1 in Patients with Hepatocellular Carcinoma. Sci Pharm.2012; 80:179-188.

34- Erinjeri JP, Contessa TTh, Constantinos T, Fleisher M, Gonen M, Sofocleous CT et al. Imageguided Thermal Ablation of Tumors Increases the Plasma Level of Interleukin-6 and Interleukin-10. J Vasc Interv Radiol 2013; 24: 1105-12.

35- Sullivan KM, Groeschl RT, Turaga KK, Tsai S, Christians KK, White SB et al. Neutrophil-toLymphocyte Ratio as a Predictor of Outcomes for Patients With Hepatocellular Carcinoma: A Western Perspective. Journal of Surgical Oncology 2014; 109: 95- 97.

36- Wang GY, Yang Y,Li H, Zhang J, Jiang N, Li MR et al. A Scoring Model Based on Neutrophil to Lymphocyte Ratio Predicts Recurrence of HBVAssociated Hepatocellular Carcinoma after Liver Transplantation. PLoS ONE 20116 (9): e25295.

37- Fondevila C, Metges JP, Fuster J, Grau JJ, Palacín A, Castells A et al. p53 and VEGF expression are independent predictors of tumour recurrence and survival following curative resection of gastric cancer. Br J Cancer. 2004; 90: 206- 15. 
38-Schaider H, Oka M, Bogenrieder, Nesbit M, Satyamoorthy K, Berking CT et al. Differential response of primary and metastatic melanomas to neutrophils attracted by IL-8. Int J Cancer 2003; 103: 335- 43.

39-Shamamian P, Schwartz JD, Pocock B J, Monea S, Whiting D, Marcus SG, Mignatti P. Activation of progelatinase A (MMP-2) by neutrophil elastase, cathepsin G, and proteinase-3: a role for inflammatory cells in tumor invasion and angiogenesis. J Cell Physiol.2001; 189: 197- 206.

40- Van Cutsem E, Arends J .: The causes and consequences of cancer-associated malnutrition. Eur J Oncol Nurs 2005; 9: S51-S63.

41-De Jong KP, Von Geusau BA, Rottier CA, Bijzet J, Limburg PC, de Vries EG et al. Serum response of hepatocyte growth factor, insulin-like growth factor-I, interleukin-6 and acute phase proteins in patients with colorectal liver metastases treated with partial hepatectomy or cryosurgery. J Hepatol 2001; 34: 422- 427.

42-Schell SR, Wessels FJ, Abouhamze A, Moldawer LL, Copeland EM 3rd. Pro- and anti inflammatory cytokine production after radiofrequency ablation of unresectable hepatic tumors. J Am Coll Surg 2002; 195: 774-781.
43-Wong VW, Yu J, Cheng AS, Wong GL, Chan HY, Chu ESet al. High serum interleukin-6 level predicts future hepato-cellular carcinoma development in patients with chronic hepatitis B. Int J Cancer 2009; 124: 2766-70.

44-Sanchez-Ortiz RF, Tannir N, Ahrar K, Wood CG. (2003): Spontaneous regression of pulmonary metastases from renal cell carcinoma after radio frequency ablation of primary tumor: an in situ tumor vaccine? .J Urol 2003;170: 178-9.

45-Rao P, Escudier B, De Baere T. Spontaneous regression of multiple pulmonary metastases after radiofrequency ablation of a single metastasis. Cardiovasc Intervent Radiol 2011;34:424- 30.

46- Dan J, Zhang Y, Peng, Z et al. Post-operative Neutrophil-to-Lymphocyte Ratio Change Predicts Survival of Patients with Small Hepato-cellular Carcinoma Undergoing Radiofrequency Ablation. PLoS ONE 2013; 8(3): e 58184.

Peer reviewers: Hamed Suliman ,Professor of Tropical Medicine and Hepatogastroenterology, Faculty of Medicine, Zagazig University, Egypt. Editor: Tarik Zaher, Professor of Tropical Medicine and Hepatogastroenterology, Faculty of Medicine, Zagazig University, Egypt 